\title{
DISKUSSION
}

\section{Die Geister, die Karlsruhe rief - eine Replik auf die Beiträge zur Wahlsystemreform in Heft 2/2010 der ZParl}

\author{
Gerd Strohmeier
}

Es ist müßig, darüber nachzudenken, ob das Bundesverfassungsgericht (BVerfG) am 3. Juli 2008, als es das Urteil zum Effekt des negativen Stimmgewichts (NSG; besser: inversen Erfolgswerts) fällte, dessen Folgen hinreichend reflektiert hat - mit anderen Worten: ob ihm bewusst war, welche Geister es damit rufen würde. Bis Mitte 2011 hat der Deutsche Bundestag das Bundeswahlgesetz zu reformieren - auch wenn dies offensichtlich schwer fällt. ${ }^{1}$ Müßig ist vor dem Hintergrund der faktischen Reformmöglichkeiten auch, darüber nachzudenken, ob die Einführung eines Mehrheitswahlsystems (zu dem auch bestimmte Formen des segmentierten Wahlsystems zu zählen sind) sinnvoll wäre. Die Mehrheitswahl ist machtpolitisch nicht (mehr) durchsetzbar - auch wenn es gute Gründe für die eine oder andere Variante dieses Wahlsystemtyps gäbe. Ernsthafte Alternativen lassen sich mittlerweile nur noch im Bereich der „strukturerhaltend-korrigierenden “ Reformen ${ }^{2}$ ausmachen - wenngleich nicht jede dieser Alternativen in gleichem Maße strukturerhaltend ist und korrigierend wirkt. Nicht alles, was verfassungsrechtlich möglich ist, muss politisch gewollt sein, und nicht alles, was politisch gewollt ist, muss verfassungsrechtlich möglich sein. Entscheidend ist, dass sich der Effekt des inversen Erfolgswerts im Korridor der verfassungsrechtlich möglichen Ausgestaltungen des Wahlsystems nicht folgenlos beheben lässt und jeder (offensichtlich auch noch so filigrane) Eingriff in das Wahlsystem zur Behebung des Effekts „Nebenwirkungen“ hat ${ }^{3}$ - die unter Umständen schwerwiegender sind als der Effekt selbst. In Heft 2/2010 der ZParl sind verschiedene Reformvorschläge diskutiert worden, die den Effekt des inversen Erfolgswerts zu beheben versuchen und dabei unterschiedliche „Nebenwirkungen“ erzeugen. Im Folgenden werden die verschiedenen Modelle systematisiert sowie deren verfassungsrechtliche und politische Konsequenzen diskutiert. $^{4}$

1 Vgl. Daniel Lübbert, Negative Stimmgewichte bei der Bundestagswahl, in: ZParl, 41. Jg. (2010), H. 2, S. $278-289$.

2 Vgl. Gerd Strohmeier, Vergangene und zukünftige Reformen des deutschen Wahlsystems, in: ders. (Hrsg.), Wahlsystemreform, Sonderband der ZPol, Baden-Baden 2009, S. $11-44$.

3 Vgl. Daniel Lübbert, a.a.O. (Fn. 1), S. 289.

4 Die Diskussion beschränkt sich auf die wesentlichen Grundzüge der Reformvorschläge und muss zwangsläufig einzelne - zur Bewertung der Reformvorschläge nicht entscheidende - Details außer Acht lassen. 


\section{Die Bandbreite für eine strukturerhaltend-korrigierende Wahlsystemreform}

Nicht wenige dürften sich angesichts der offensichtlichen Probleme bei der möglichst folgenlosen Behebung des Effekts des inversen Erfolgswerts insgeheim wünschen, das BVerfG hätte dem Gesetzgeber keine Wahlsystemreform auferlegt, sondern entschieden, dass der Effekt - wie Überhangmandate im Allgemeinen - als eine „notwendige Folge des besonderen Charakters der personalisierten Verhältniswahl" ${ }^{\text {"5 }} \mathrm{zu}$ betrachten und verfassungsrechtlich nicht zu beanstanden ist - vielleicht mit Ausnahme des Auftretens bei Nachwahlen, durch die er instrumentalisierbar werden kann und eine besondere Problematik entfaltet. ${ }^{6}$ Die Karlsruher Richter haben aber nun einmal entschieden, dass „\$7 Absatz 3 Satz 2 in Verbindung mit $\$ 6$ Absätze 4 und 5 des Bundeswahlgesetzes [...] die Grundsätze der Gleichheit und der Unmittelbarkeit der Wahl [verletzt], soweit hierdurch ermöglicht wird, dass ein Zuwachs an Zweitstimmen zu einem Verlust an Sitzen der Landeslisten oder ein Verlust an Zweitstimmen zu einem Zuwachs an Sitzen der Landeslisten führen kann"7, obwohl es „zuvor - und in voller Kenntnis des bereits im Jahre 1997 in dem von der Landesregierung Niedersachsen geführten Normenkontrollverfahren gerügten und thematisierten NSG - entschieden beziehungsweise dem Bundestag in Berichterstatterschreiben signalisiert hatte, dass die NSG verfassungskonform seien "8. Das Gericht hat sogar entschieden, dass der Effekt des inversen Erfolgswerts den Grundsatz der Gleichheit der Wahl „in eklatanter [!] Weise"9) verletzt, obwohl - wie Joachim Behnke ${ }^{10}$ richtig erkennt - der Effekt zum Teil eine Angleichung der Erfolgswertgleichheit bedeutet: wenn mehr Stimmen zu weniger Sitzen führen, nicht aber, wenn weniger Stimmen zu mehr Sitzen führen. Interessanterweise hat das BVerfG in seinem Urteil vom 3. Juli 2008 auch - im Einklang mit seinem äußerst knapp gefällten Urteil $1997^{11}$ - deutlich gemacht, dass Überhangmandate per se nicht verfassungswidrig sind, obwohl die Existenz beziehungsweise die Möglichkeit des Entstehens von Überhangmandaten zusammen mit Listenverbindungen die Voraussetzung für das Entstehen des Effekts des inversen Erfolgswerts bildet und Überhangmandate auch unabhängig davon - aufgrund ihrer realen Disproportions- und potenziellen Mehrheitsef-

5 Vgl. BVerfG, 2 BvF 1/95 vom 10. April 1997.

6 Das Bundesverfassungsgericht hätte durchaus „den leichten Ausweg eines Nachwahlverbots“ (Franz Urban Pappi / Michael Herrmann, Überhangmandate ohne negatives Stimmgewicht: Machbarkeit, Wirkungen, Beurteilung, in: ZParl, 41. Jg. (2010), H. 2, S. 260 - 278, S. 261) beschreiten können. Schließlich könnte eine Instrumentalisierung des Effekts des inversen Erfolgswerts - wie auch andere ungewollte Begleiterscheinungen von Nachwahlen - durch ein Nachwahlverbot weitestgehend vermieden und das Nachwahlverbot (durch die Aufstellung von Ersatzkandidaten) relativ leicht umgesetzt werden.

7 BVerfG, 2 BvC 1/07 vom 3. Juli 2008.

8 Heinrich Lang, A-Drs. 16(4)592 F: 3.

9 BVerfG, a.a.O. (Fn. 7).

10 Vgl. Joachim Behnke, Überhangmandate und negatives Stimmgewicht: Zweimannwahlkreise und andere Vorschläge, in: ZParl, 41. Jg. (2010), H. 2, S. 247 - 260.

11 Dabei hat das Bundesverfassungsgericht die grundsätzliche Existenz von Überhangmandaten (ohne Verrechnung oder Ausgleich) als „notwendige Folge des besonderen Charakters der personalisierten Verhältniswahl mit der Wahlgleichheit nach Art. 38 Abs. 1 Satz 1 GG [für] vereinbar" erklärt, also verfassungsrechtlich nicht beanstandet - allerdings den Gesetzgeber angehalten, darauf zu achten, „dass sich die Zahl der Überhangmandate in Grenzen hält“, BVerfG, a.a.O. (Fn. 5). 
fekte bei der Regierungsbildung - kritikwürdig sind. ${ }^{12}$ Entscheidend ist aber, dass inverse Erfolgswerte nicht ohne Überhangmandate, aber Überhangmandate ohne inverse Erfolgswerte möglich sind. ${ }^{13}$

Dass Überhangmandate (ohne Ausgleich) verfassungsrechtlich gerechtfertigt werden können, heißt aber noch lange nicht, dass sie politisch gewollt sein müssen. Politisch beziehungsweise politikwissenschaftlich kann man jedenfalls nicht oder nur schwerlich für Überhangmandate eintreten, indem man ihnen - wie Franz Urban Pappi und Michael Herrmann $^{14}$ - die Funktion zuschreibt, als „Mehrheitsboni [...] zur Erleichterung der Regierungsbildung" zu fungieren. Schließlich können Überhangmandate unter anderem als „Bonus“ für besonders erfolglose Parteien auftreten, knappe Mehrheiten destabilisieren oder Minderheiten in Mehrheiten transformieren. Natürlich entstehen Überhangmandate - neben anderen Ursachen ${ }^{15}$ - durch Stimmensplitting im Rahmen des „Koalitionswählens“. Sie aber - wie Pappi und Herrmann ${ }^{16}$ - als "Mehrheitsbonus zugunsten des Parteilagers [...], das sich besser koordinieren kann", zu verstehen, greift eindeutig zu weit - nicht zuletzt auch deshalb, weil es im gegenwärtigen Fünf-Fraktionen-Parlament kaum mehr klare Koalitionsoptionen gibt. Wenn sich Überhangmandate überhaupt rechtfertigen lassen, dann allenfalls als Bonus für Parteien, die in der Lage sind, Direktmandate (in größerem Umfang) zu gewinnen. ${ }^{17}$ Die Suche nach überzeugenden Gründen für die Existenz von Überhangmandaten ist also politisch ebenso schwierig wie verfassungsrechtlich. Überhangmandate können letztlich nicht gerechtfertigt, sondern nur in Kauf genommen werden politisch wie verfassungsrechtlich: als „notwendige Folge des besonderen Charakters der personalisierten Verhältniswahl"18 beziehungsweise "geringstes Übel“ (im Vergleich zu Nachteilen, die Ausgestaltungsformen der personalisierten Verhältniswahl ohne Überhangmandate aufweisen). Dass - wie Pappi und Herrmann ${ }^{19}$ argumentieren - „Beurteilungskriterien einer reinen Verhältniswahl“, das heißt „in einem nationalen Wahlkreis ohne Sperrklausel“ (gegen die Überhangmandate in der Tat „eine Petitesse“ sind), für das deutsche Wahlsystem „unangemessen sind“, ist zwar kein Hauptargument für Überhangmandate, wohl aber eine Abschwächung des Hauptarguments gegen sie. Man muss das deutsche Wahlsystem nicht - wie Pappi und Herrmann ${ }^{20}$ - aufgrund des „Mischwahlcharakter[s] in der Legitimationskette", entgegen der sinnvollen und breit akzeptierten Einordnung Dieter Nohlens ${ }^{21}$, als Mischwahlsystem begreifen, um Abweichungen vom Grundsatz der Erfolgswertgleichheit rechtfertigen zu können. Entscheidend für die Einteilung als Verhältniswahlsystem ist nicht die Frage, ob, sondern $z u$ welchem Grad dieses eine Stimmen-Mandats-

12 Vgl. Eckhard Jesse, Demokratie in Deutschland: Diagnosen und Analysen, Köln 2008, S. 246 ff.

13 Vgl. Joachim Behnke, a.a.O. (Fn. 10), S. 247.

14 Franz Urban Pappi / Michael Herrmann, a.a.O. (Fn. 6), S. 271.

15 Vgl. zu den Entstehungsursachen von Überhangmandaten: Florian Grotz, Die personalisierte Verhältniswahl unter den Bedingungen des gesamtdeutschen Parteiensystems. Eine Analyse der Entstehungsursachen von Überhangmandaten seit der Wiedervereinigung, in: PVS, 41. Jg. (2000), H. 4, $707-729$.

16 Franz Urban Pappi / Michael Herrmann, a.a.O. (Fn. 6), S. 272.

17 Ebenda, S. 276.

18 BVerfG, a.a.O. (Fn. 5).

19 Franz Urban Pappi / Michael Hermann, a.a.O. (Fn. 6), S. 263, S. 270.

20 Ebenda, S. $270 \mathrm{f}$.

21 Vgl. Dieter Nohlen, Wahlrecht und Parteiensystem: Zur Theorie und Empirie der Wahlsysteme, Opladen 2007, S. 351. 
Proportionalität hervorbringt. So kann kein (Verhältnis-)Wahlsystem dieser Welt eine absolute Stimmen-Mandats-Proportionalität garantieren. Zudem verfolgen die meisten Verhältniswahlsysteme neben der Erfolgswertgleichheit - als primäres Repräsentationsziel - auch das Ziel, über (künstliche oder natürliche Hürden) die Funktionsfähigkeit und Stabilität der Regierung beziehungsweise des gesamten politischen Systems zu sichern.

Die Überhangmandate stehen zwar nicht im Mittelpunkt der Bundesverfassungsgerichtsentscheidung zum Effekt des inversen Erfolgswerts, wohl aber im Zentrum der Diskussion zu dessen Behebung: Während sie für Behnke ${ }^{22}$ das „eigentliche Problem“ darstellen und grundsätzlich vermieden werden müssen, sind sie nach Pappi und Herrmann ${ }^{23}$ „bewusst und nicht nur als unschöne[r] Nebeneffekt“ zu erhalten. An dieser Stelle ist zwischen zwei Typen von Überhangmandaten zu unterscheiden: internen und externen. Externe Überhangmandate treten bereits bei der Oberverteilung, das heißt bei der Verteilung der Mandate auf die Wahlvorschläge auf Bundesebene, auf. Sie entstehen also, wenn ein Wahlvorschlag auf Bundesebene mehr Direktmandate erhält, als ihm auf der Grundlage der Zweitstimmen Mandate zustehen. Bei der Bundestagswahl 2009 wurden der CSU als erster deutscher Partei drei externe Überhangmandate zugeteilt. Die CDU hätte die Schwelle zum Gewinn externer Überhangmandate beinahe auch überschritten, errang aber letztlich bundesweit exakt so viele Direktmandate, wie ihr auf der Grundlage der Zweitstimmen Mandate zustanden. ${ }^{24}$ Interne Überhangmandate treten erst bei der Unterverteilung, das heißt bei der Verteilung der auf eine Listenverbindung entfallenen Mandate auf die einzelnen Landeslisten dieser Verbindung, auf. Sie entstehen also, wenn eine Landesliste einer Listenverbindung mehr Direktmandate erhält, als ihr auf der Grundlage der Zweitstimmen in diesem Land Mandate zustehen. Die dabei entstehenden Überhangmandate sind der Regelfall - und können zum Effekt des inversen Erfolgswerts führen.

Vor diesem Hintergrund wäre es irreführend, die aktuellen Beiträge zur Wahlsystemreform in ein Lager, das Überhangmandate bewahrt, und ein Lager, das Überhangmandate verhindert, zu spalten. Schließlich haben alle der in Heft 2/2010 der ZParl präsentierten Reformvorschläge - unabhängig davon, ob deren Vertreter Überhangmandate (als „eigentliches Problem“) grundsätzlich vermeiden oder („bewusst und nicht nur als unschönen $\mathrm{Ne}$ beneffekt“) prinzipiell (zu einem gewissen Grad) erhalten möchten - gemeinsam, dass sie interne Überhangmandate zur Behebung des Effekts des inversen Erfolgswerts verhindern müssen und (wie noch gezeigt wird) externe Überhangmandate zum Erhalt „des besonderen Charakters der personalisierten Verhältniswahl“ nicht gänzlich verhindern (sondern allenfalls nachträglich ausgleichen oder korrigieren) können. Sinnvoller (aber möglicherweise unspektakulärer) ist es, die aktuellen Beiträge zur Wahlsystemreform wie folgt einzuteilen: in ein Lager, das an Listenverbindungen festhält, und ein Lager, das auf Listenverbindungen (und damit das zweistufige, aus Ober- und Unterverteilung bestehende Mandatsvergabesystem) verzichtet. Entlang dieser beiden grundlegenden Reformvarianten gruppieren sich die gegenwärtig vorherrschenden Positionen - in der Politikwissenschaft sowie offensichtlich auch in der Politik. ${ }^{25}$

22 Joachim Behnke, a.a.O. (Fn. 10), S. 247.

23 Franz Urban Pappi / Michael Herrmann, a.a.O. (Fn. 6), S. 266.

24 Vgl. Daniel Lübbert, a.a.O. (Fn. 1), S. 282 ff.

25 Vgl. Katja Wilke, Das Tückische beim Zählen der Stimmen, http://www.das-parlament.de/2010/ 41-42/Innenpolitik/31782689.html (Abruf am 27. November 2010). 


\section{Lösung mit Listenverbindungen}

Ein Reformvorschlag, der an Listenverbindungen festhält, lässt sich als „Überhangmandate-Kompensationsmodell" deklarieren. Dieses Modell, das in unterschiedlichen Varianten $^{26}$ vorgeschlagen wurde und den ,am meisten diskutierte[n] Vorschlag ${ }^{227}$ darstellt, sieht die Verrechnung der (internen) Überhangmandate mit Listenmandaten in anderen Ländern ${ }^{28}$ beziehungsweise die Verrechnung der Direktmandate mit den Listenmandaten bei der Oberverteilung vor. ${ }^{29}$ Das Überhangmandate-Kompensationsmodell verhindert den Effekt des inversen Erfolgswerts, funktioniert aber nur dann reibungslos, wenn man einen Zwang zur Verbindung der Landeslisten einer Partei einführt ${ }^{30}$, und weist trotz seiner allgemeinen Beliebtheit „einige gravierende Mängel auf "31. So führt es - wie auch das BVerfG ${ }^{32}$ erkannte - zu dem (zwar nicht verfassungsrechtlichen, aber doch politischen) Problem, dass es den föderalen Proporz, das heißt den partei-internen Länderproporz, beeinträchtigt. Im derzeit geltenden Wahlsystem tun dies Überhangmandate natürlich auch, allerdings in Form einer „Draufgabe“ zum proportional zustehenden Anteil an Mandaten. Dabei kann eine Partei in einem Land nicht hinter den Anteil zurückfallen, der ihr auf der Grundlage der Zweitstimmen zusteht, während sie nach dem Überhangmandate-Kompensationsmodell nur nicht hinter den Anteil zurückfallen kann, den sie durch Direktmandate gewonnen hat: „Der frühere Überhangbonus einzelner Landesverbände wird zu einem merklichen Malus für jene Landesverbände, die für die Mandate bezahlen müssten, die nach geltendem Wahlrecht Überhänge schaffen. "33 Dies kann dazu führen, dass Parteien in einzelnen Ländern, wie zum Beispiel die CDU in Bremen, überhaupt keinen Abgeordneten mehr stellen. ${ }^{34}$ Zudem werden durch das Überhangmandate-Kompensationsmodell nicht - wie Friedrich Pukelsheim ${ }^{35}$ behauptet - alle, sondern nur interne Überhangmandate verhindert. Externe können weiterhin entstehen und bestimmte Parteien - gegenwärtig die CSU und wohl auch die CDU - privilegieren. Will man dies verhindern, muss man auf Regelungen zurückgreifen, die entweder - wie die Vergabe von Ausgleichsmandaten - den Bundestag deutlich ,aufblähen“ oder - wie die Nicht-Vergabe von den Direktmandaten der betroffenen Partei mit dem geringsten prozentualen Erststimmenergebnis ${ }^{36}$ - äußerst unelegant, verfassungsrechtlich fragwürdig und politisch problematisch sind. ${ }^{37}$

26 Eine davon bildet der gegen Ende der letzten Wahlperiode eingebrachte und gescheiterte Gesetzentwurf zur Wahlsystemreform der Fraktion Bündnis 90/Die Grünen. Vgl. BT-Drs. 16/11885.

27 Joachim Behnke, a.a.O. (Fn. 10), S. 259.

28 Vgl. ebenda, S. 251.

29 Vgl. Franz Urban Pappi / Michael Herrmann, a.a.O. (Fn. 6), S. 262.

30 Vgl. Joachim Behnke, a.a.O. (Fn. 10), S. 256; Franz Urban Pappi / Michael Herrmann, a.a.O. (Fn. 6), S. 275.

31 Ebenda, S. 259.

32 Vgl. BVerfG, a.a.O. (Fn. 7).

33 Franz Urban Pappi / Michael Herrmann, a.a.O. (Fn. 6), S. 276.

34 Vgl. ebenda, S. 265.

35 Vgl. Friedrich Pukelsheim, Bundeswahlgesetz - Nächste Etappe, in: DVBL, 123. Jg. (2008), H. 14, S. $889-897$.

36 Vgl. Hans Meyer, Lösungsmöglichkeiten nach dem Wahlrechtsurteil des BVerfG vom 3. Juli 2008, in: DVBL, 124. Jg. (2009), H. 3, S. $137-146$.

37 Vgl. Joachim Behnke, a.a.O. (Fn. 10), S. 255 f.; Franz Urban Pappi / Michael Herrmann, a.a.O. (Fn. 6), S. 265. 
Ein weiterer Reformvorschlag, der an Listenverbindungen festhält, lässt sich als „Zweipersonenwahlkreis-Modell“ etikettieren. Dieses Modell, das unlängst von Behnke ${ }^{38}$ vorgeschlagen wurde, sieht vor, dass die Direktmandate in Zweipersonenwahlkreisen vergeben werden, die Wähler dabei weiterhin nur eine Erststimme haben und pro Wahlkreis der Kandidat mit den meisten und der mit den zweitmeisten Stimmen gewählt ist. Folglich würden die Direktmandate nicht mehr nach den Grundsätzen des bei der britischen Parlamentswahl Anwendung findenden First-Past-The-Post-Systems, sondern nach den Prinzipien des früher bei der japanischen Parlamentswahl praktizierten Single-Non-TransferableVote-Systems vergeben werden. Das Zweipersonenwahlkreis-Modell hat einen gewissen Charme, jedoch - neben anderen Mängeln - einen vernichtenden Nachteil: Es vermeidet den Effekt des inversen Erfolgswerts nicht. Es mag mit einer geringeren Wahrscheinlichkeit zu dem Effekt führen, kann ihn aber ebenso wenig ausschließen wie das Auftreten von (internen und externen) Überhangmandaten. Selbst auf der Grundlage von Behnkes wohlwollender (das heißt zweitstimmenbasierter) Simulationsrechnung lässt sich der Effekt des inversen Erfolgswerts nachweisen. Zwar käme es - in diesem Beispiel - in keinem Land zu Überhangmandaten, doch stünden in einigen Bundesländern dort antretenden verbundenen Landeslisten exakt so viele Mandate zu, wie die jeweilige Partei Direktmandate gewonnen hat. Bei den betroffenen Landeslisten - der SPD im Saarland und in Schleswig-Holstein, der CDU in Bremen und Mecklenburg-Vorpommern sowie der Linken in Sachsen und Thüringen ${ }^{39}$ - zöge kein Kandidat auf diesem Weg in den Bundestag ein und wäre die Voraussetzung für den Effekt des inversen Erfolgswerts gegeben. Dass dieser nicht nur möglich, sondern auch eintreten würde, zeigt eine Zusammenschau mit der Berechnung von Daniel Lübbert ${ }^{40}$ : Hätte die CDU in Mecklenburg-Vorpommern 47.930 Stimmen weniger erhalten, müsste sie einen Sitz an die CDU in Niedersachsen abgeben. Dabei würde diese faktisch ein Mandat gewinnen (da ein weiterer Bewerber über die Landesliste in den Bundestag einziehen könnte), die CDU in Mecklenburg-Vorpommern aber faktisch keinen Sitz verlieren (da das verlorengegangene Mandat als Überhangmandat erhalten bliebe). Infolgedessen hätten weniger Stimmen zu mehr Mandaten - und das Zweipersonenwahlkreis-Modell zum Effekt des inversen Erfolgswerts geführt.

\section{Lösung ohne Listenverbindungen}

Ein Reformvorschlag, der auf Listenverbindungen verzichtet, lässt sich als „Landeswahlkreis-Modell“ deklarieren. Dieses Modell, das unlängst von Pappi und Herrmann ${ }^{41}$ vertreten wurde, sieht vor, dass die Länder (wie 1949) geschlossene Wahlkreise bilden und damit keine Verbindung mehr zwischen den Landeslisten einer Partei besteht. Damit wird der Effekt des inversen Erfolgswerts verhindert - und zudem dafür gesorgt, dass die Verteilung der Abgeordneten auf die Länder nicht mehr, wie nach dem derzeit geltenden Wahlsystem,

38 Vgl. ebenda, S. 257 ff.

39 Vgl. ebenda, S. 259; Bundeswahlleiter, Endgültige Sitzverteilung nach Ländern, in: http://www. bundeswahlleiter.de/de/bundestagswahlen/BTW_BUND_09/veroeffentlichungen/engueltige/ voetab3.pdf (Abruf am 27. November 2010).

40 Vgl. Daniel Lübbert, a.a.O. (Fn. 1), S. 283.

41 Vgl. Franz Urban Pappi / Michael Herrmann, a.a.O. (Fn. 6), S. 262. 
von der Wahlbeteiligung beeinflusst wird. Die Fünf-Prozent-Klausel kann in dem Landeswahlkreis-Modell entweder auf die Bundes- oder die Landesebene bezogen werden. Pappi und Herrmann ${ }^{42}$ entscheiden sich gegen die zuletzt genannte Variante (die eine Verrechnung der Stimmen auf Bundesebene obsolet machen würde), obwohl sie sich zum Beispiel bei der Bundestagswahl 2009 nicht anders ausgewirkt hätte und möglicherweise mehr der Logik dieses Modells entspricht. Allerdings bestünde bei der Anwendung auf Landesebene grundsätzlich die Gefahr, dass das Parteiensystem (weiter) zersplitterte, kleine bundesweit (klar) unter der Fünf-Prozent-Hürde liegende (extreme) Parteien in manchen Ländern in den Bundestag einzögen, aber kleine bundesweit (deutlich) über der Fünf-Prozent-Hürde liegende Parteien in manchen Ländern von der Mandatsverteilung ausgeschlossen wären, und die Herausbildung „starker unabhängiger Landesverbände angeregt“ würde. Folglich erscheint es - primär um einer „Schwächung des nationalen Parteiensystems vorzubeugen " 43 - durchaus sinnvoll, die Sperrklausel auf die Bundesebene zu beziehen. Dies würde im Rahmen des Landeswahlkreis-Modells keineswegs - wie Behnke $e^{44}$ argumentiert - „eine Inkonsistenz bedeuten“, sondern könnte - neben der Vermeidung der Probleme bei der Anwendung der Sperrklausel auf Landesebene - sogar als Ausdruck des viel beschworenen „unitarischen Charakters" des Bundestags gesehen werden. Ein Widerspruch zum „unitarischen Charakter" des Bundestags wäre das Landeswahlkreis-Modell - wie Lübbert ${ }^{45}$ in Anlehnung an Hans Meyer ${ }^{46}$ behauptet - aber grundsätzlich nicht, zumindest kein wesentlich größerer als die nach dem derzeit geltenden Wahlsystem praktizierte Aufstellung und Wahl von Landeslisten. Auch die - von Meyer befürchteten - zentrifugalen Kräfte im Parteiensystem dürften kaum größer sein als im gegenwärtigen Wahlsystem.

Das Landeswahlkreis-Modell ermöglicht - bewusst - die Entstehung von Überhangmandaten, müsste aber keineswegs - wie Behnke ${ }^{47}$ vermutet - dazu führen, dass die Anzahl der Überhangmandate im Vergleich zum bestehenden Wahlsystem ansteigt. Pappi und Herrmann zeigen sogar, dass das Landeswahlkreis-Modell - aufgrund des Wegfalls bestimmter Ursachen von Überhangmandaten (wie der ungleichen Wahlbeteiligung) - weniger Überhangmandate als das geltende Wahlsystem hervorbringen kann. ${ }^{48}$ Sicherlich könnte sich - wie Behnke ${ }^{49}$ einwirft - der Anreiz zu strategischem Stimmensplitting - und damit auch die Anzahl an Überhangmandaten - tendenziell erhöhen. Ein „Wettlauf um Überhangmandate " 50 ist jedoch ebenso wenig zu erwarten wie derzeit. Das Landeswahlkreis-Modell würde durch die Wahlkreisziehung - wie Pappi und Herrmann zeigen - mit wenigen Ausnahmen (Bremen und Saarland) auch nicht zu einer Benachteiligung kleiner Parteien führen. Außerdem könnten durch die Anwendung der Fünf-Prozent-Klausel auf Bundesebene, die Größe der (meisten) Wahlkreise und das angewandte Stimmenverrechnungsverfahren (Sainte-Laguë / Schepers) erhebliche Verzerrungen mit Blick auf die Vertretungszahlen der (kleinen) Parteien vermieden werden.

42 Vgl. ebenda, S. 266 ff.

43 Ebenda, S. 268.

44 Joachim Behnke, a.a.O. (Fn. 10), S. 249.

45 Vgl. Daniel Lübbert, a.a.O. (Fn. 1), S. 287.

46 Vgl. Hans Meyer, a.a.O. (Fn. 36), S. $137-146$.

47 Vgl. Joachim Behnke, a.a.O. (Fn. 10), S. 250.

48 Vgl. Franz Urban Pappi / Michael Herrmann, a.a.O. (Fn. 6), S. 272.

49 Vgl. Joachim Behnke, a.a.O. (Fn. 10), S. 250.

50 Franz Urban Pappi / Michael Herrmann, a.a.O. (Fn. 6), S. 277. 
Ein weiterer Reformvorschlag, der auf Listenverbindungen verzichtet, lässt sich als „Bundeslisten-Modell“ etikettieren. Dieses Modell, das dem „unitarischen Charakter“ des Bundestags vollends gerecht wird und in der aktuellen Diskussion - zu Unrecht - unterbelichtet ist, sieht die Schaffung von Bundeslisten vor. Das Bundeslisten-Modell ermöglicht das Entstehen von Überhangmandaten in dem Maße, wie das bestehende Wahlsystem externe Überhangmandate ermöglicht, und verhindert den Effekt des inversen Erfolgswerts ebenso wie das Landeswahlkreis-Modell. So wie letzteres allerdings in einem diskrepanten Verhältnis zum „unitarischen Charakter“ des Bundestags gesehen wird, ließe sich das BundeslistenModell in einem gewissen Spannungszustand zum „föderalen Charakter“ der Bundestagswahl sehen (der bislang durch die Wahl von Landeslisten zum Ausdruck kam). Ein substanzieller Nachteil des Bundeslisten-Modells besteht in diesem Zusammenhang darin, dass die Parteien im Zuge der Listenaufstellung auf den regionalen Proporz achten müssten - was allerdings zu einem deutlich besseren partei-internen Länderproporz als beim Überhangmandate-Kompensationsmodell führen dürfte beziehungsweise durch entsprechende Vorgaben bei der Listenaufstellung gewährleistet werden könnte. Zudem ist aber auch zu berücksichtigen, dass alle Patreien - auch CSU und CDU - zwangsläufig im gesamten Bundesgebiet zur Wahl stünden, was sicherlich nicht unmaßgebliche, im Moment aber nicht vollständig absehbare Folgen für das Parteiensystem hätte.

\section{Ein Plädoyer für einen Verzicht auf Listenverbindungen}

Das Überhangmandate-Kompensationsmodell ist offensichtlich - wie die Wissenschaftlichen Dienste des Deutschen Bundestags dokumentieren - „in den Augen vieler Experten eine ideale Lösung für das Problem der negativen Stimmgewichte" ${ }^{\text {" } 1}$. Angesichts der doch gravierenden Nachteile dieser Lösung ist es jedoch äußerst erfreulich, dass sich nicht alle Experten dem festgestellten Mainstream angeschlossen haben. Das ÜberhangmandateKompensationsmodell ist nicht (oder bestenfalls aus einer bestimmten, stark eingeschränkten Perspektive) - wie Lübbert ${ }^{52}$ behauptet - die „minimal-invasive Lösung mit den bisher vermutlich geringsten Nebenwirkungen“. Das von Behnke vorgeschlagene Zweipersonenwahlkreis-Modell ist indessen überhaupt keine Lösung, da es den Effekt des inversen Erfolgswerts nicht vermeidet. Um die Struktur des bestehenden Wahlsystems bestmöglich zu erhalten ${ }^{53}$ und dessen Mechanismen mit Gewissheit um den Effekt des inversen Erfolgswerts zu korrigieren, ohne dabei allzu große und ungewünschte Nebenwirkungen zu erzeugen, erscheint eine Wahlsystemreform empfehlenswert, die auf Listenverbindungen verzichtet. Das von Pappi und Herrmann vorgeschlagene Modell ist sicherlich nicht „das Beste aus den Welten von Mehrheits- und Verhältniswahl“ "54, aber möglicherweise - neben dem zu Unrecht vernachlässigten Bundeslisten-Modell - das Geeignetste, um am bestehenden (Verhältniswahl-)System festzuhalten und den Effekt des inversen Erfolgswerts zu beheben.

51 Daniel Lübbert, Negative Stimmgewichte und die Reform des Bundestags-Wahlrechts, in: Deutscher Bundestag, Wissenschaftliche Dienste, Info-Brief WD 8 - 3000 - 020/09 vom 18. März 2009, S. 20.

52 Ders., a.a.O. (Fn. 1), S. 289.

53 Vgl. Franz Urban Pappi / Michael Herrmann, a.a.O. (Fn. 6), S. 277.

54 Ebenda, a.a.O. (Fn. 6), S. 278. 